\title{
Effect of Fine Particle Peening (FPP) Conditions on Microstructural Characteristics of Ti-6Al-4V Alloy *
}

\author{
Yutaka KAMEYAMA **and Jun KOMOTORI *** \\ ** RIKEN (The Institute of Physical and Chemical Research), 2-1, Hirosawa, Wako, Saitama, 351-0198, \\ Japan \\ (Old address: Keio University, 3-14-1, Hiyoshi, Kohoku-ku, Yokohama, Kanagawa, 223-8522, Japan) \\ E-mail:ykame@2002.jukuin.keio.ac.jp \\ *** Keio University, 3-14-1, Hiyoshi, Kohoku-ku, Yokohama, Kanagawa, 223-8522, Japan
}

\begin{abstract}
The microstructure of Ti-6Al-4V alloy treated with Fine Particle Peening (FPP) was studied. The influence of different peening times, particle supply rates, and particle sizes on the microstructure was especially investigated. FPP treatment using steel particles created a modified microstructure with a lamellar feature beside the treated surface. The modified structure showed higher hardness comparing to the interior region and it was accompanied by Fe elements transferred from particles. Energy Dispersive X-ray Spectroscopy (EDS) analyses revealed that the amount of transferring Fe elements was increased as peening times and/or particle supply rates were increased. Fe transfer was also accelerated by FPP treatment using smaller particles. The reasons for this behavior were as follows: (1) plastic deformation induced by collisions of particles was effectively accumulated with an increase of peening time and particle supply rate, and (2) plastic deformation induced by collisions of particles became more severe as particle size decreased. The accumulation and severity of plastic deformation strongly affected the formation of the modified microstructure enriched with transferred elements.
\end{abstract}

Key words: Peening, Surface Modification, Metal Transfer, Diffusion

\section{Introduction}

Fine Particle Peening (FPP) treatment, where the surfaces of materials are bombarded with fine particles, is widely applied in various industries because of its beneficial effects on improving fatigue properties of metallic materials ${ }^{(1),(2)}$. Moreover, the changes in the microstructure by FPP treatment have recently been reported. Umemoto et. al. indicated that nanocrystallization was induced by FPP treatment because of severe plastic deformation of treated materials ${ }^{(3)}$. The authors reported that particle elements were concentrated on the FPP-treated surface ${ }^{(4)}$. The authors have also demonstrated the advantages of the surface with particle elements to improve adhesion of DLC coating ${ }^{(5)}$, to reduce toxicity of biomaterials ${ }^{(6)}$, and so on.

Despite a noticeable potential of the FPP treatment to create the microstructure containing particle elements, detailed reasons for microstructural change have not been clarified. A proposed reason is the diffusion of particle elements ${ }^{(7)}$. In this assumption, diffusion is accelerated by the temperature rising on the treated surface. However, there is no experimental evidence that proves the temperature rising is caused by FPP treatment. Therefore, it is necessary to investigate the details of the microstructural change induced by 
the FPP treatment.

The present study evaluated the characteristics of modified microstructure by the FPP treatment. Then the effect of FPP conditions on the modified microstructure formation was described. The influences of peening times, particle supply rates, and particle sizes on the microstructure were specifically focused upon.

\section{Experimental procedure}

Ti-6Al-4V alloy discs with a $15 \mathrm{~mm}$ diameter and a $4 \mathrm{~mm}$ thickness were used as substrates. FPP treatment was performed with an air suction type apparatus (SFK-2S type, Fuji manufacturing Co. Ltd.) in which SKH59 steel particles were bombarded from the peening nozzle to the treated material. The distance between nozzle and specimen was $100 \mathrm{~mm}$, peening pressure was $0.6 \mathrm{MPa}$, and peening angle (the angle between the nozzle and the specimen surface) was $90^{\circ}$. Peening time range was from $0.1 \mathrm{sec}$ to $600 \mathrm{sec}$, particle supply rate was $0.3 \mathrm{~g} / \mathrm{sec}, 1 \mathrm{~g} / \mathrm{sec}, 2.5 \mathrm{~g} / \mathrm{sec}$, and $10 \mathrm{~g} / \mathrm{sec}$, and nominal particle size was $\phi 50 \mu \mathrm{m}$ (diameter varied $\phi 43 \sim 65 \mu \mathrm{m}$ ) and $\phi 195 \mu \mathrm{m}$ (diameter varied $\phi 180 \sim 212 \mu \mathrm{m}$ ). For comparison, conventional steel shots with a diameter of $\phi 800 \mu \mathrm{m}$ were also used. The influences of different peening times, particle supply rates, and particle sizes on the microstructure were compared. Peening time was controlled by the particle shutter apparatus when peening time was smaller than $5 \mathrm{sec}$. The particle shutter apparatus was constructed by combining a rotating disc with a slit, a DC motor, and a gear unit. As shown in Fig.1, that was placed between the peening nozzle and a specimen. The distance between the peening nozzle and the rotating disc was $50 \mathrm{~mm}$. Particles hit the specimen only when the slit comes below the peening nozzle. Particle supply rate was controlled by an electromagnetic linear feeder.

The surface and the cross section of each specimen were observed and analyzed by a scanning electron microscope (SEM) to investigate microstructural changes after the FPP treatment. Hardness measurement was carried out by a micro Vickers hardness tester on the tapered cross sections (tapered angle: $4.4^{\circ}$ ) in order to evaluate the characteristics of the modified microstructure. Presence or absence of the particle elements (Fe) were identified by an energy dispersive X-ray spectroscopy (EDS) analyses on the treated surface. Fe concentration on the FPP-treated surface was quantitatively measured with EDS, as an averaged value of 5 areas (each measurement point was approximately $700 \times 700 \mu \mathrm{m}$ ).

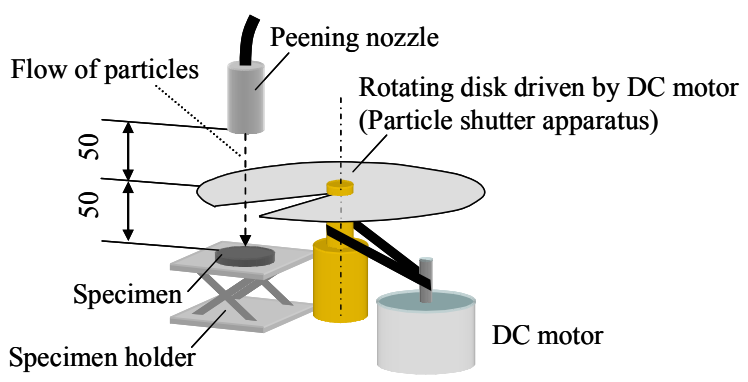

Fig.1 Schematic illustration of FPP treatment setup 


\section{Results and discussion}

\subsection{The effect of FPP treatment on microstructure}

After $15 \mathrm{sec}$ of FPP treatment with particle supply rate of $10 \mathrm{~g} / \mathrm{sec}$ and particle size of $\phi 50 \mu \mathrm{m}$, the surface of a specimen was completely covered by rough texture. It indicated that all areas of the surface were treated uniformly. Figure 2(a) shows a typical feature of microstructure on the cross section of the specimen with the FPP treatment. Lamellar featured microstructure is observed beside the surface. Figures 2(b), (c) and (d) show the magnified image, the EDS images of $\mathrm{Ti}$, and that of $\mathrm{Fe}$ corresponding to the squared region in Fig.2(a), respectively. Figure 2(d) indicates the presence of Fe elements in the lamellar featured microstructure. Fe elements concentrated in the titanium substrate are supplied from steel particles during FPP treatment.

Forming a heterogeneous microstructure that is described above is attributed to severe plastic deformation during FPP process. Plastic deformation enhances the metal transfer from particle to the treated surface and/or embedment of crushed particles. Then those are mechanically mixed into the substrate, creating the Fe-rich lamellar microstructure ${ }^{(8)}$. In this process, temperature of the surface is risen by collisions of particles. This possibly enhances the mechanical mixing process. In addition, Takagi .et.al. reported that nanocrystallization was induced by FPP treatment ${ }^{(9)}$. Increase of grain boundaries due to nanocrystallization could enhance diffusion of the particle elements from mixed fragments to the substrate microstructure. Consequently, the lamellar featured microstructure accompanied by particle elements may be formed owing to the transferring, mixing and diffusion of particle elements.
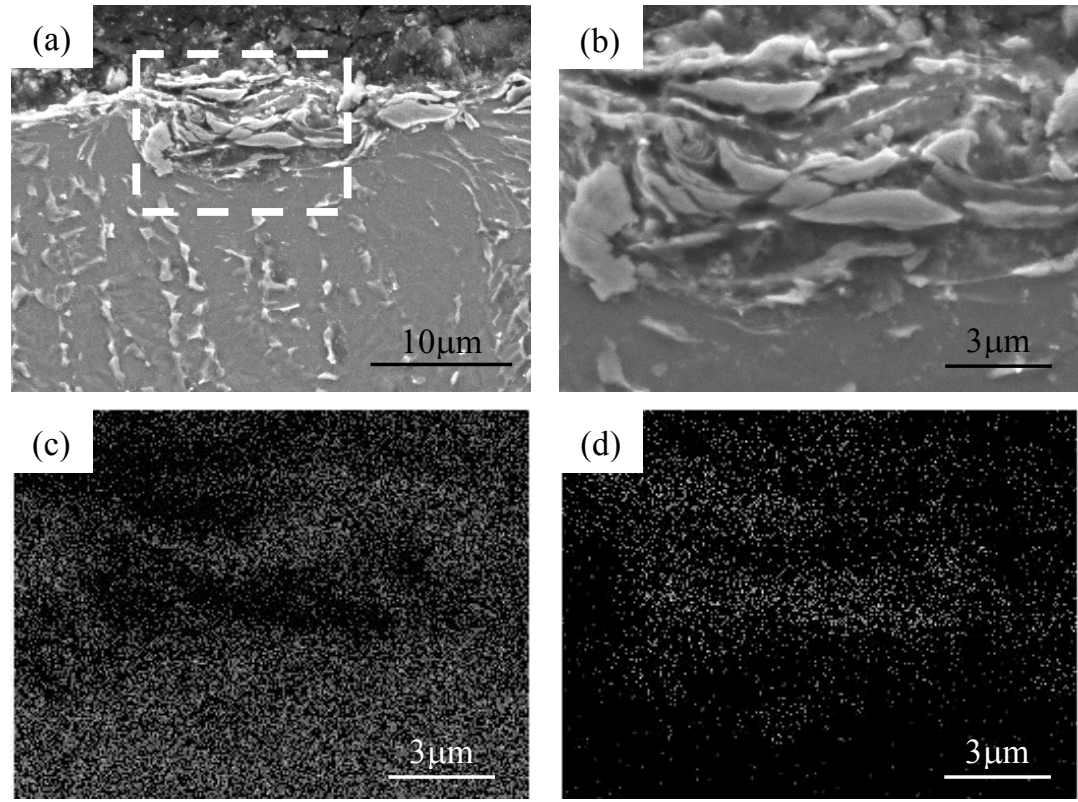

Fig.2 Cross sectional SEM observation and EDS analyses of FPP-treated specimen with a peening time of $15 \mathrm{sec}$. (a) SEM image of microstructure, (b) Magnified SEM image of squared region in (a),

(c) EDS Ti Ka image corresponding to (b), and (d) EDS Fe K $\alpha$ image corresponding to (b) 


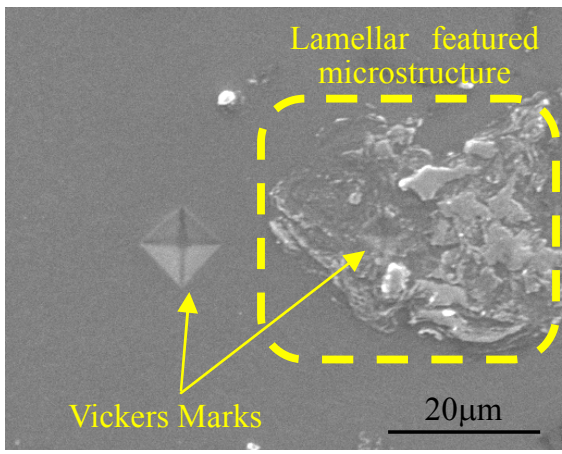

Fig.3 Typical feature of Vickers indentations (On the tapered cross section)

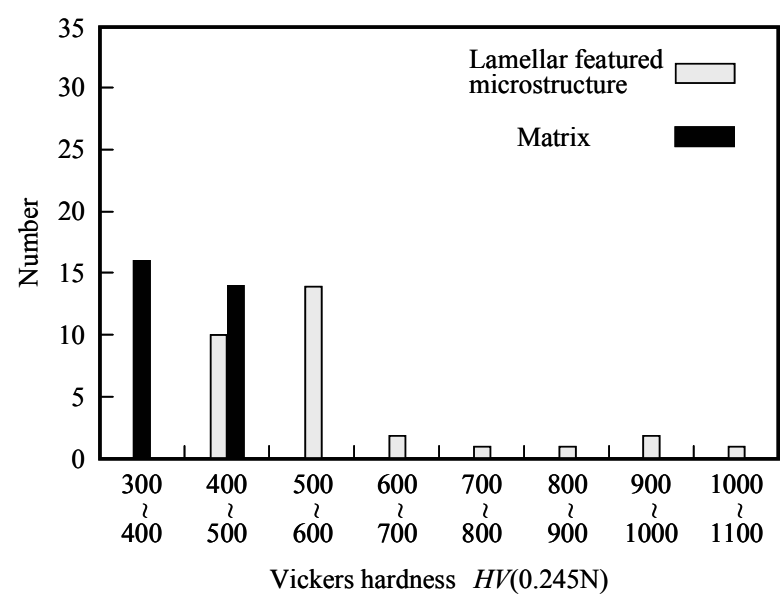

Fig.4 Histogram of hardness on the lamellar microstructure and the matrix

A microstructure enriched with particle elements is observed on the other specimens prepared with the other FPP conditions. This implies that microstructural changes occur under various particle supply rates and/or particle sizes. The effect of FPP conditions on the formation of the modified microstructure will be discussed in $\S 3.2$.

In order to examine the detailed characteristics of the lamellar featured microstructure, hardness of the work hardened region just beside the surface was measured on the tapered cross section. Figure 3 shows the typical feature of Vickers indentations on the lamellar microstructure and the non-lamellar microstructure (matrix). The lamellar microstructure exhibits a higher hardness than the matrix. Figure 4 shows a histogram comparing the hardness on the lamellar microstructure and the matrix. The hardness of the lamellar one becomes significantly higher than that of the matrix. The lamellar microstructure involves mixed particle fragments. The hardness of steel particles (approximately $800 \sim 1000 \mathrm{HV}$ ) is relatively higher than that of the substrate so that the hardness of the lamellar region increases. Mixed fragments are finely crushed and then concentrated, so that the most of hardness values of the lamellar region is not as high as the original particles. Some of hardness data, which show noticeably higher value, are affected by scattering of hardness values of the particles. Other possible reason for hardening is severe plastic deformation, inducing mechanical recrystallization in the deformed region. Because of work hardening as well as decrease of grain size, the hardness of lamellar region is increased.

As we discussed, lamellar-featured microstructure created by FPP is accompanied by Fe elements and exhibits high hardness. Thus, formation of the lamellar microstructures possibly improves the surface characteristics such as wear resistance, fatigue properties and so on, although those microstructures are locally formed and not covering the surface uniformly. 


\subsection{The influence of FPP conditions on transferring of particle elements}

Figure 5 compares the relationship between peening time and $\mathrm{Fe}$ element concentration measured on the treated surface with a particle supply rate of $0.3 \mathrm{~g} / \mathrm{sec}, 1 \mathrm{~g} / \mathrm{sec}$, and $2.5 \mathrm{~g} / \mathrm{sec}$ (particle size: $\phi 50 \mu \mathrm{m}$ ). The amount of Fe elements, which are transferred from particles and then mixed into substrates, increases as peening time increases. Figure 5 also indicates that the increasing rates of transferred amount become larger with an increase of particle supply rates.

Figure 6 compares the relationship between peening time and Fe element concentration on the treated surface with a particle size of $\phi 50 \mu \mathrm{m}, \phi 195 \mu \mathrm{m}$ and $\phi 800 \mu \mathrm{m}$ (particle supply rate: $0.3 \mathrm{~g} / \mathrm{sec}$ ). In this case, the increasing rate of Fe concentrations is logarithmically decreased with an increase of particle size.

In the same peening time, the number of particles colliding on the surface becomes larger with an increase of particle supply rate and/or a decrease of particle size. The results described above indicate that the transferred amount of Fe is affected by the number of particles collisions. The formation of the modified microstructure accompanied by transferred elements is enhanced by the "degree of accumulation" of particles collisions.

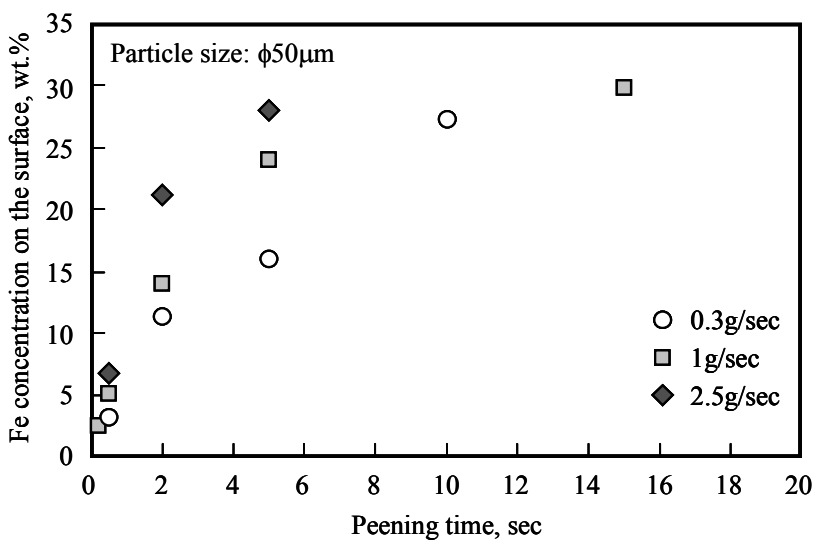

Fig. 5 Surface Fe concentration as a function of peening time for a variation of particle supply rate

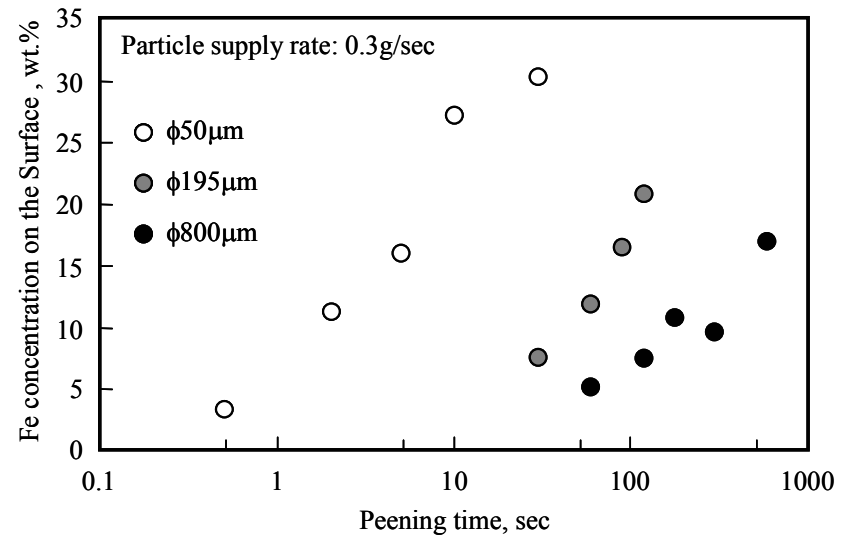

Fig.6 Surface Fe concentration as a function of peening time for a variation of particle size 


\subsection{Dominant factor affecting the transferring behavior during FPP treatment}

In Figs. 5 and 6, the authors compared the relationship between peening time and transferred $\mathrm{Fe}$ amount among the specimens with different FPP conditions. It is shown that transferring of $\mathrm{Fe}$ is enhanced as the particles collisions become accumulated. In above discussion, the number of particles collision differs depending on particle supply rates and particle sizes even if the peening time is the same. In order to compare the degree of accumulation of particle collision among the different FPP conditions, the coverage is introduced in this study. The coverage is calculated as follows.

$$
C(\%)=\frac{t}{t_{\mathrm{FC}}} \times 100
$$

Where $C$ is the coverage, $t$ is peening time, and $t_{\mathrm{FC}}$ is the full coverage time, in which original polished surface is uniformly covered with collision dents. $t_{\mathrm{FC}}$ varies depending on the particle supply rates and the particle sizes. The amount of particles bombarded in FPP treatment is steadily controlled, so that the coverage is equal to the ratio of the numbers of particles bombarded in $t$ and in $t_{\mathrm{FC}}$. Considering this, the coverage can represent the degree of accumulation of particle collision.

Then, $t_{\mathrm{FC}}$ is estimated with the following procedure. Optical microscopic observation was carried out on the center of a specimen in order to estimate the ratio of area on which dents were formed. Figure 7(a) shows a typical microscopic image. Then the microscopic image was binarized (see Fig.7(b)) and the ratio of dent-formed area was calculated. The ratio of dent-formed area increases with an increase of peening time in the earlier stage of FPP treatment (see Fig.7(c)). Then the ratio reaches a stable value implying that the surface is uniformly covered by collision dents. The peening time when the ratio of dent-formed area becomes steady is defined as $t_{\mathrm{FC}}$. Figure 8 shows $t_{\mathrm{FC}}$ estimated on the specimens treated with each FPP condition.

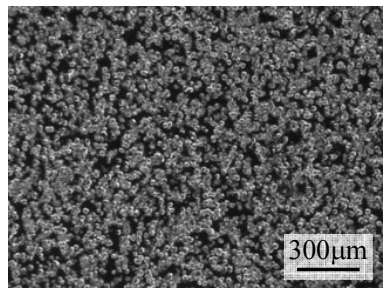

(a) Optical microscopic image of FPP-treated surface

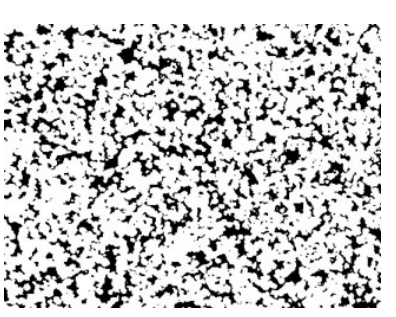

(b) Binarized image of (a)

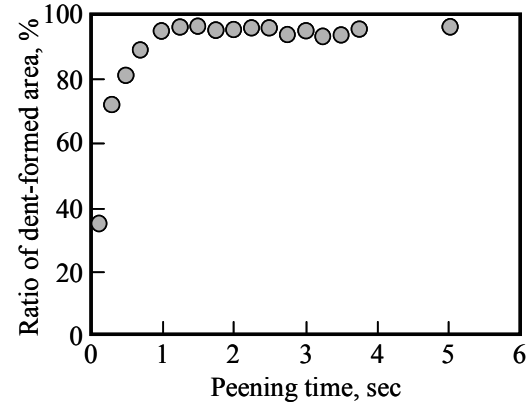

(c) Relationship between the ratio of dent-formed area and peening time

Fig.7 Evaluation of dent-formed area to estimate $t_{\mathrm{FC}}$
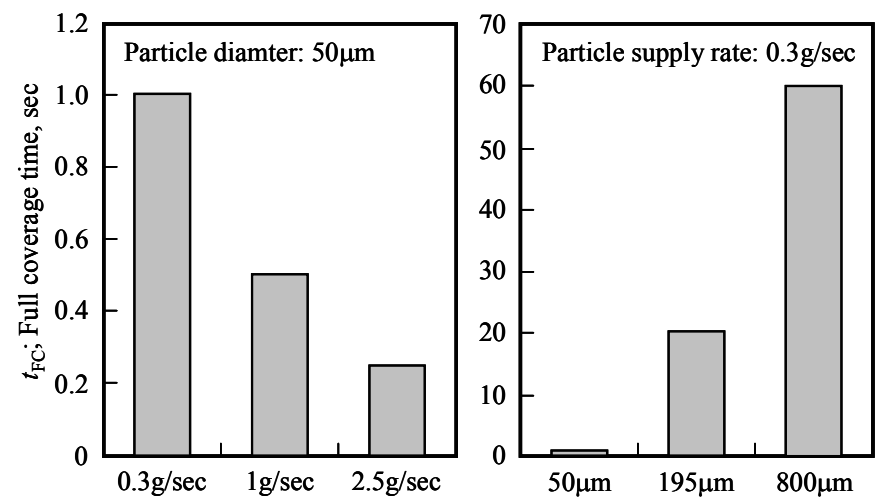

Fig. $8 t_{\mathrm{FC}}$; full coverage time 


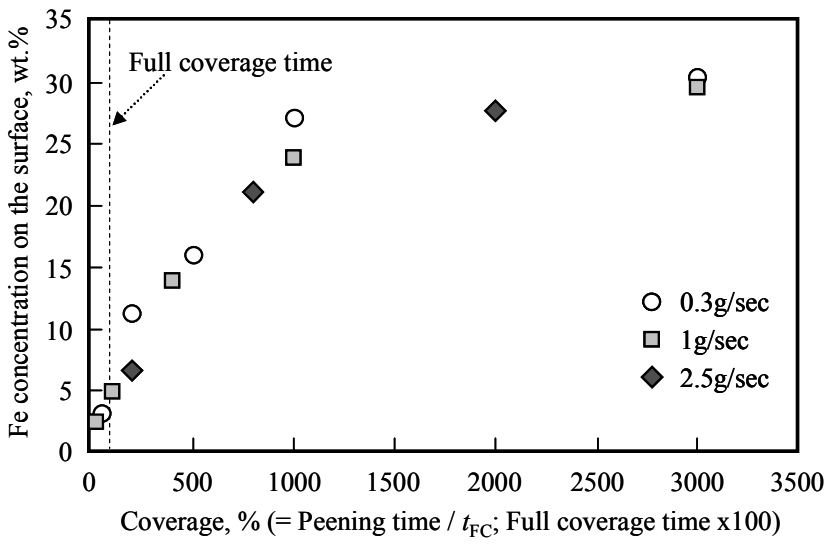

Fig.9 Relationship between surface Fe concentration and coverage (Particle size: $\phi 50 \mu \mathrm{m}$ )

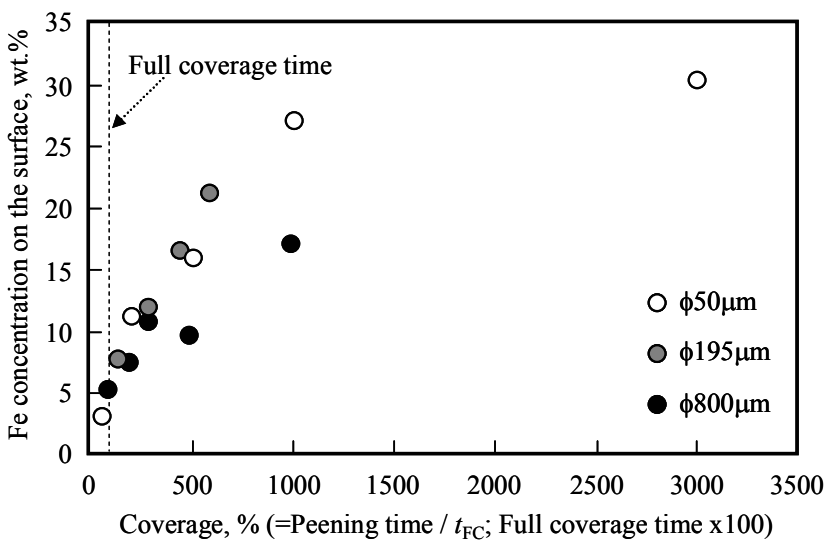

Fig.10 Relationship between surface Fe concentration and coverage (Particle supply rate: $0.3 \mathrm{~g} / \mathrm{sec}$ )

Then replacement of peening time into the coverage is carried out for Figs. 5 and 6. Figure 9 shows relationships between the coverage and the transferred amount for the variation of a particle supply rate. When the coverage is introduced, the increasing rate of the transferred amount becomes almost similar among the different particle supply rates. Indicated as above, the coverage represents the degree of accumulation of particle collision. The result shown in Fig.9 means that the transferring of particle elements is enhanced as the plastic deformation induced by collisions of each particles accumulates.

On the other hand, Fig.10 shows relationships between the coverage and transferred amount for the variation of a particle size. In this case, the increasing rate of transferred amount is higher in cases of smaller particles $(\phi 50 \mu \mathrm{m}$ and $\phi 195 \mu \mathrm{m})$ than that of larger ones $(\phi 800 \mu \mathrm{m})$ which is usually applied for a conventional shot peening method. This result means that transferring of particle elements is effectively accelerated when smaller particles are used.

Because the transferring behavior results from plastic deformation during the FPP treatment, the relationship between particle size and the transferring behavior is attributed to the severity of plastic deformation. In order to discuss the plastic deformation behavior, the simple estimation is carried out as shown below.

When a particle is colliding on the treated surface, the treated area is indented by the particle (see Fig.11). At an indentation depth $z$, the equation of motion of the particles colliding on the treated surface vertically can be described as

$$
\frac{4}{3} \pi r^{3} \rho \frac{d v}{d t}=-\pi a^{2} \bar{p}
$$


Where, $r$ is the averaged diameter of particles, $\rho$ is density of particle material, $v$ is velocity of particles at the time of $t$, and $a$ is the radius of the dent correlating with indentation depth $z . \quad \bar{p}$ is an averaged contact pressure.

Considering the geometric relationship,

$$
a^{2}+(r-z)^{2}=r^{2}
$$

If $z$ is very small, $a$ can be described as

$$
a \approx \sqrt{2 r z}
$$

$\bar{p}$ can be estimated from the yielding stress of the material as described below ${ }^{(10)}$.

$$
\bar{p}=3 \sigma_{y}
$$

Here, depth of collision dent $h_{\mathrm{d}}$ can be assumed by integrating of Eq.(2),

$$
\begin{gathered}
\frac{4}{3} \pi r^{3} \rho \int_{V}^{0} v d v=-6 \pi r \sigma_{y} \int_{0}^{h_{\mathrm{d}}} z d z \\
h_{\mathrm{d}}=\left(\frac{2 \rho}{9 \sigma_{y}}\right)^{\frac{1}{2}} r V
\end{gathered}
$$

Where, $V$ is particle velocity before collision. Equation (7) indicates that depth of dent is proportional to particle velocity and particle size.

The relationship between the plastic zone formed below a dent and the depth of collision dent is calculated by Al-Obaid ${ }^{(11)}$. The depth of plastic zone $h_{\mathrm{p}}$ can be expressed as

$$
\frac{h_{\mathrm{p}}}{r}=2.57\left(\frac{h_{\mathrm{d}}}{r}\right)^{\frac{1}{2}}
$$

Substituting Eq.(7) into Eq.(8), $h_{\mathrm{p}}$ is described as follows.

$$
\frac{h_{\mathrm{p}}}{r}=1.76\left(\frac{\rho}{\sigma_{y}}\right)^{\frac{1}{4}} V^{\frac{1}{2}}
$$

Equation (9) describes the ratio of the depth of plastic zone $h_{\mathrm{p}}$ versus a particle size $r$ as a function of particle velocity.

It is known that the smaller particle size makes an impact velocity greater ${ }^{(12)}$. Thus, the ratio $h_{\mathrm{p}} / r$ gets greater when finer particles are used for FPP treatment. This implies that FPP treatment with finer particle size can effectively introduce severe plastic deformation.

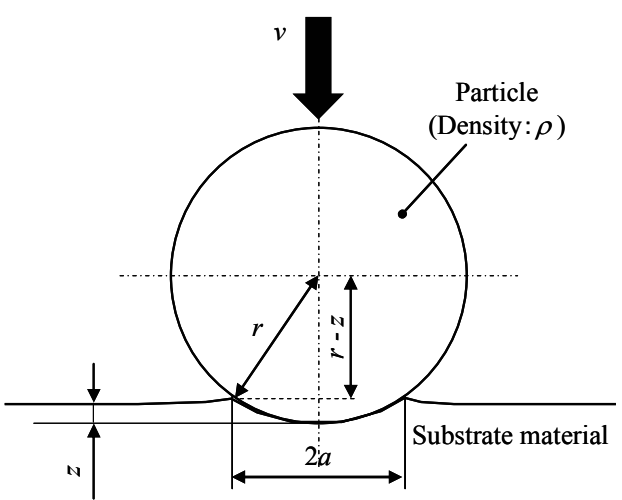

Fig.11 Schematic illustration of indentation of a particle corresponding to indentation depth of $\mathrm{z}$ 


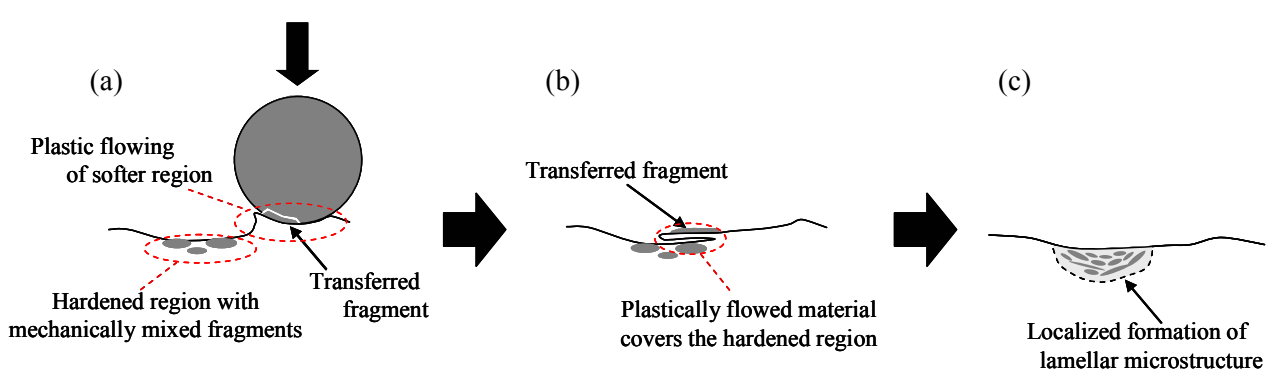

Fig.12 Schematic illustration of localized formation of lamellar microstructure (a) Particle collision on the adjacent area of hardened region. (b) Covering by the flowed material. Transferred fragment is brought onto the hardened region.

(c) Lamellar microstructure formation resulted from repeated covering.

Plastic deformation is a driving force of transferring and/or embedding of particle fragments in the FPP process. More severe plastic deformation possibly enhances these phenomena. In the earlier stage of FPP treatment, the treatment with finer particles effectively accelerates the transferring, embedding and mechanical mixing behavior of particle elements, because plastic deformation is more severe.

As we discussed in section 3.1, the microstructure with mixed particle fragments is relatively harder than the matrix. Difference in the hardness might affect the microstructural changing. When hardened region is locally created by mixing of the fragments, it is easier for the matrix material to be plastically deformed than for the hardened one. A flowed material of softer matrix, which is accompanied with transferred fragment, covers the hardened region as shown in the schematic illustration of Fig.12. As a result, the particle fragments are locally concentrated on the covered region. As these behaviors repeatedly occur, a layered microstructure is created. In addition, transferring and/or embedding of particles might be suppressed on the hardened region because of the lack of plastic deformation. Figures 9 and 10 indicate that the increase in Fe concentration becomes slower as the coverage increases. The reason for this behavior is spreading of hardened region beside the surface with an increase of the coverage.

As we discussed, plastic deformation is important to cause the transferring behavior. Two dominant factors which affect transferring behavior are demonstrated. One is the accumulation of plastic deformation. Plastic deformation induced by particle collisions could be effectively accumulated with an increase in the number of particles. This means that FPP treatment has an advantage to enhance transferring behavior because this treatment can bombard a huger amount of particles comparing to a conventional shot peening method. The other is the severity of plastic deformation. FPP treatment can introduce more severe plastic deformation, so that transferring behavior becomes accelerative. Because of these advantages, FPP treatment can successfully create the modified microstructure, which is accompanied by particle elements as well as shows excellent mechanical properties such as a high hardness.

\section{Conclusion}

The characteristics of the microstructure modified by Fine Particle Peening (FPP) treatment were investigated. With a special focus on transferring from particles, the influences of different peening time, particle supply rate, and particle size on microstructure were discussed. The transferred elements from particles are concentrated in the microstructure that exhibits a lamellar feature and higher hardness comparing to the internal one. The reason for creating the microstructure is mechanical mixing of transferred 
fragment induced by severe plastic deformation during FPP process. The amount of transferred elements is increased with an increase of peening time. The amount of transferred elements becomes larger with an increase of particle supply rate. Increasing rate of the transferred amount becomes almost the same when peening time is replaced into the coverage, which relates the number of particles colliding on the treated surface. This indicates that the formation of lamellar microstructure with transferred elements is affected by the accumulation of plastic deformation induced by collisions of a particle. The amount of transferred elements also increases when the smaller particles are chosen. The reason for this behavior is that plastic deformation becomes much severe with a decrease of particle size. The accumulation and severity of plastic deformation are the dominant factors which affect the formation of modified microstructure enriched with transferred elements.

\section{References}

(1) D. Yonekura, J. Noda, J. Komotori, M. Shimizu and Y. Miyasaka, The fatigue properties of low carbon Cr-Mo steel modified with $\mathrm{WPC}^{\circledR}$ process, The proceedings of the second international conference on advanced materials development and performance evaluation and application, Vol.1, (1999), pp.64-67

(2) J. Sugishita, N. Egami, I. Usami, M. Yamada, Y. Miyasaka and K. Nakashima, Effects of WPC treatment on surface strength of metals, Proceedings of the $10^{\text {th }}$ International Conference on Surface Modification Technologies, Vol.10, (1997), pp.594-606

(3) M. Umemoto, Y. Todaka and K. Tsuchiya, Formation on nanocrystalline structure in steels by air blast shot peening, Materials Transactions, Vol.44, (2003), pp.1488-1493

(4) Y. Kameyama, J. Komotori and E. Shimodaira, Diffusion induced by fine particles bombardment (FPB) treatment, Journal of Material Testing Research Association of Japan, Vol.48, No.4, (2003), pp.241-244

(5) Y. Kameyama and J. Komotori, Tribological properties of structural steel modified by fine particle bombardment (FPB) and diamond-like carbon hybrid surface treatment, Wear, Vol.263, (2007), pp.1354-1363

(6) K. Murai, J. Komotori, Y. Kameyama, T. Yamada and E. Shimodaira, Biocompatibility of Ti-6Al-4V alloy modified by fine particle bombardment (FPB), Proceedings of 10th World Conference on Titanium Ti-2003, Vol.5, (2003), pp.3362-3367

(7) H. Ogiwara, Modification of piston sliding surface for internal combustion engine by fine particle peening of solid lubricant, Journal of Japanese Society of Tribologist (In Japanese), Vol.47, (2002), pp.895-900

(8) Y. Kameyama, A. Sasago and J. Komotori, Material transfer and diffusion in peening process on Ti-6Al-4V alloy, Proceedings of 11th World Conference on Titanium Ti-2007, Vol.2, (2007), pp.1719-1722

(9) S. Takagi, M. Kumagai, Y. Ito, S. Konuma and E. Shimodaira, Surface nanocrystallization of carburized steel JIS-SCr420 by fine particle peening, The journal of the Iron and Steel Institute of Japan (In Japanese), Vol.92, No.5 (2006), pp.318-326

(10) D. Tabor, The Hardness of Metals, (2000), Clarendon Press

(11) Y. F. Al-Obaid, A rudimentary analysis of improving fatigue life of metals by shot-peening, Transactions of ASME Journal of Applied Mechanics, Vol.57, (1990), pp.307-312

(12) K. Ogawa, T. Asano, A. Saito, K. Kawamura, M. Ogino and H. Aihara, Measurement and analysis of shot velocity in pneumatic shot peening, Transactions of the Japan Society of Mechanical Engineers C (In Japanese), Vol.60, No.571, (1994), pp.1120-1125 